\title{
SUMO-specific protease 2 in Mdm2-mediated regulation of p53
}

\author{
M Jiang ${ }^{1}$, S-Y Chiu ${ }^{1,2}$ and W Hsu ${ }^{*, 1}$
}

Genetic analysis in mice has revealed a key genetic pathway, SUMO-specific protease 2 (SENP2)-Mdm2-p53, essential for trophoblast development. Targeted disruption of SENP2 impairs the G-S transition required for mitotic and endoreduplication cell cycles during the expansion of trophoblast stem cells and their differentiation into polyploidy cells, respectively. The disruption disturbed the subcellular distribution and SUMO modification of Mdm2, leading to interference with p53 degradation. Here, we further explore the mechanism underlying SENP2-mediated regulation of Mdm2 in p53-induced cellular stress. We identify a specific isoform of SENP2 necessary and sufficient to negatively regulate the p53-dependent transcription and its related stress responses. This isoform-specific effect is attributed to the differential compartmentalization of SENP2. SUMO conjugation of Mdm2 induces its co-localization and association with SENP2 in promyelocytic leukemia bodies. Biochemical studies show that SENP2 catalyzes the desumoylation process of Mdm2. SENP2-dependent regulation of Mdm2 is sensitive to its p53-binding activity. Our findings led us to propose a mechanism underlying the SENP2-mediated regulation of Mdm2 that is critical for genome integrity in p53-dependent stress responses.

Cell Death and Differentiation (2011) 18, 1005-1015; doi:10.1038/cdd.2010.168; published online 24 December 2010

Small ubiquitin-related modifier (SUMO, also known as sentrin) is a member of the ubiquitin-like modifier family. ${ }^{1}$ This covalent conjugation process is reversible and highly evolutionarily conserved among species. ${ }^{2}$ SUMO functions in a manner similar to ubiquitin, where it is bound to target proteins as part of a post-translational modification system. However, unlike ubiquitination with a well-established role in targeting protein degradation, SUMO modification is involved in a variety of cellular processes, including protein trafficking, transcriptional regulation, cell survival and death, and protein stability. ${ }^{3}$ Many sumoylated proteins have been shown to preferentially accumulate in specific complexes such as the nuclear pore and promyelocytic leukemia (PML) bodies., ${ }^{4,5}$ Similar to ubiquitination, sumoylation requires processing, conjugation and transfer. The transfer process, which covalently conjugates SUMO polypeptides to their targets, is catalyzed by E3 ligases. ${ }^{6}$ The reverse desumoylation process is mediated by SUMO-specific proteases (SENPs). Although SENPs have been linked to catalyze SUMO modification in various physiological systems, their roles in mammalian development and pathogenesis remain largely elusive.

We previously demonstrated that SENP2 is essential for modulating the p53/Mdm2 circuit in trophoblast development. $^{7}$ Targeted disruption of SENP2 in mice impaired the G-S transition required for mitotic and endoreduplication cell cycles during expansion of trophoblast stem (TS) cells and their differentiation into polyploid cells, respectively. ${ }^{7}$ The SENP2 ablation apparently disturbed the subcellular distribution and SUMO modification of Mdm2, ${ }^{7}$ which is a ubiquitin E3 ligase essential for targeting p53 degradation. ${ }^{8}$ Although the functional consequences of sumoylation have been difficult to elucidate, both p53 and Mdm2 could be modified by the SUMO pathway. ${ }^{9,10}$ Nonetheless, we were able to show that reintroducing SENP2 into the mutants alleviates the defects by reducing the sumoylation of $\mathrm{Mdm} 2$, thereby diminishing the p53 level. ${ }^{7}$ Downregulation of p53 also alleviated the SENP2null phenotypes, while stimulation of p53 caused abnormalities resembling those of the SENP2 mutants. ${ }^{7}$ Using gain-offunction and loss-of-function analyses, our genetic evidence revealed a key genetic pathway, SENP2-Mdm2-p53, pivotal for genome replication underlying cell proliferation and differentiation.

The involvement of SENP2 in the Mdm2-mediated regulation of p53 prompted us to further investigate the mechanistic details on this regulatory pathway. As p53 stabilization is a crucial step for its nuclear accumulations, ${ }^{11}$ SENP2 may be involved in p53-dependent aneuploidy, genome instability and tumorigenesis. ${ }^{12-14} \mathrm{~A}$ recent report showed that the repression of SENPs induces p53-dependent premature senescence, ${ }^{15}$ suggesting that SENP2 may have a role in genotoxic stress. In this study, we found that SENP2 is necessary and sufficient to negatively regulate p53-dependent transcription

\footnotetext{
${ }^{1}$ Department of Biomedical Genetics, Center for Oral Biology, James P Wilmot Cancer Center, University of Rochester Medical Center, 601 Elmwood Avenue, Box 611, Rochester, NY, USA

*Corresponding author: W Hsu, Department of Biomedical Genetics, Center for Oral Biology, James P Wilmot Cancer Center, University of Rochester Medical Center, 601 Elmwood Avenue, Box 611, Rochester, NY 14642, USA. Tel: 585275 7802; Fax: 585276 0190; E-mail: wei_hsu@urmc.rochester.edu

${ }^{2}$ Current address: Dana-Farber Cancer Institute, Boston, MA, USA

Keywords: SENP2; SUMO; sumoylation; PML; apoptosis; stress

Abbreviations: DAPI, 4'-6-diamidino-2-phenylindole; ECL, electrochemiluminescence; GFP, green fluorescent protein; GST, glutathione S-transferase; HA, hemagglutinin; MT, myc tag; NEM, N-ethylmaleimide; NES, nuclear export signal; NLS, nuclear localization sequence; PML, promyelocytic leukemia; SDS-PAGE, sodium dodecyl sulfate-polyacrylamide gel electrophoresis; SENP, SUMO-specific protease; SUMO, small ubiquitin-related modifier; TS, trophoblast stem; TUNEL, terminal deoxynucleotidyl transferase dUTP nick end labeling

Received 04.5.10; revised 15.11.10; accepted 16.11.10; Edited by M Oren; published online 24.12.10
} 
through modulation of protein stability. SENP2 also modulates the stress responses mediated by $\mathrm{p53}$. Although SENP2 encodes three proteins generated by alternative splicing, we identified an isoform-specific effect on the p53 regulation and its related responses. This was attributed to the differential compartmentalization of SENP2 isoforms modulating its interaction with Mdm2. Biochemical analysis further showed that SENP2 not only associates with Mdm2 but also modulates its SUMO conjugation. Our findings led to a proposed mechanism underlying the SENP2-mediated regulation of p53 critical for genome integrity in stress responses.

\section{Results}

Isoform-specific regulation of p53 by SENP2. We investigated whether the regulation of p53 by SENP2 results in alterations of p53-dependent transcription. In the SENP2-null cells, the level of p53 was enhanced (Figure 1a), accompanied by elevation of p53-mediated transcriptional activity (Figure 1b). Because the genetic mutation inactivated all three gene products of SENP2 generated by alternative splicing, ${ }^{7}$ we next determined if the effect of SENP2 on p53 is regulated by a specific isoform (Figure 1i). High levels of SENP2 (Figure 1c), but not the other two forms, SENP2-M (Figure 1e) and SENP2-S (Figure 1g), drastically diminished the cellular levels of $\mathrm{p} 53$. We also observed a slight reduction caused by SENP2-S. In addition, p53-dependent transcription is sensitive only to SENP2 overexpression (Figure 1d). The expression of SENP2-M (Figure 1f) and SENP2-S (Figure 1h) did not significantly alter gene transcription dependent upon p53. Using gain-of-function and loss-of-function analyses, the results indicated that SENP2 is necessary and sufficient for modulating p53 level, as well as p53-dependent transcription. Furthermore, there is an isoform-specific regulation of p53 by SENP2.

SENP2 modulates p53-mediated stress response. The isoform-specific effect of SENP2 on p53 led us to examine the DNA damage responses sensitive to the loss of p53. It has been shown that p53 deficiency affects cell survival caused by DNA damage-induced apoptosis. ${ }^{16,17}$ Indeed, doxorubicin treatment at high concentration $(1 \mu \mathrm{M})$ resulted in the death of HCT116 cells (Figure 2a). The doxorubicinmediated cell survival problem was alleviated in HCT116 cells with the p53 deletion (Figure 2a). To further elucidate the regulation of p53 by SENP2 and determine the role of this regulatory pathway in cell stress, we established a number of stably transformed cell clones expressing different isoforms of SENP2 (Figure 2b). Similar to the earlier observation (Figure 1), high levels of SENP2 $(n=24)$, but not SENP2-M $(n=12)$ and SENP-S $(n=14)$, repressed the cellular levels of p53 (Figure 2b). None of the derivatives of HCT116 $(n=26)$, expressing either SENP2-M or SENP2-S, showed alterations of the p53 level. In contrast, 16 out of 24 HCT116SENP2 cell lines displayed significant reduction of p53 and were selected for functional analyses.

We first examined whether the DNA damage-induced apoptosis is sensitive to the expression of SENP2 isoforms. In HCT116-SENP2, but not HCT116-SENP2M and a

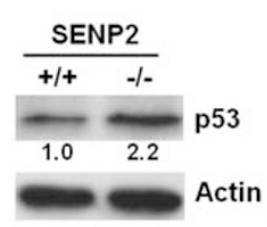

C

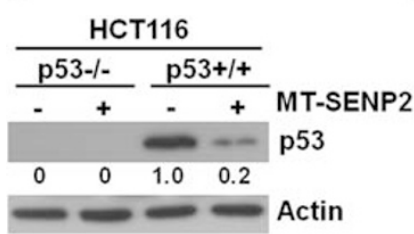

b

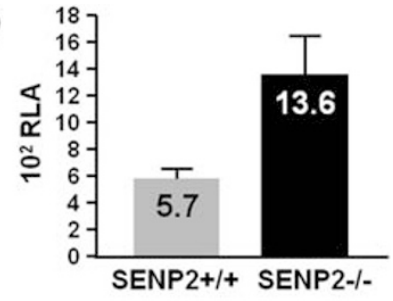

d

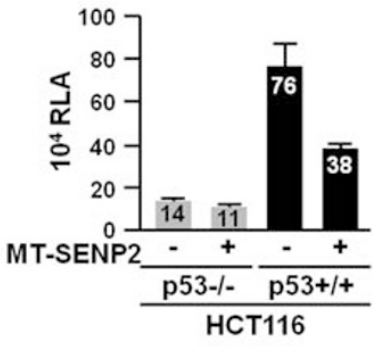

e

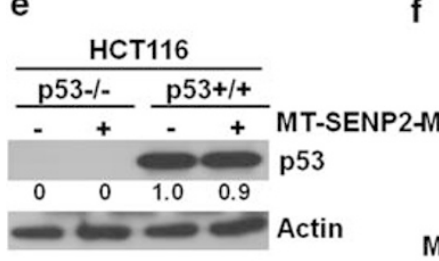

f

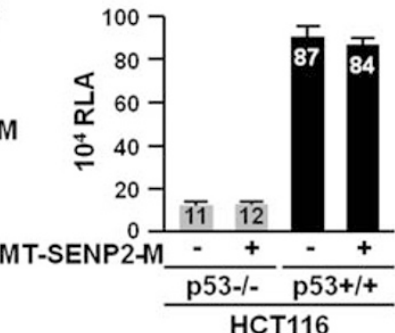

g

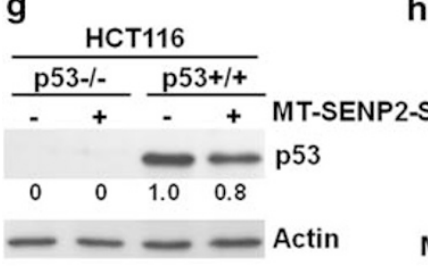

h

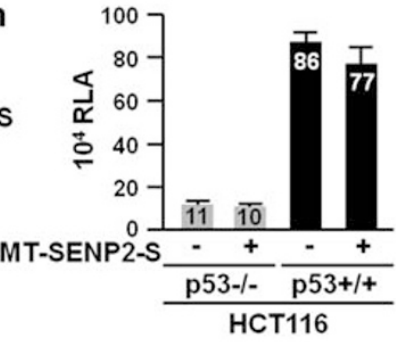

Figure 1 Isoform-specific regulation of p53 by SENP2. (a) Immunoblot analysis shows the p53 level elevated by genetic inactivation of SENP2 in TS cells. (b) The loss of SENP2 induces enhancement of the p53-dependent transcription. The protein level of $p 53$ is downregulated by high levels of SENP2 (c), but not SENP2-M (e) and SENP2-S (g) isoforms. Immunoblot analysis of p53 was performed in HCT116 colon cancer cells containing knockout (p53-l-) and wild-type p53 $(\mathrm{p} 53+/+)$, with $(+)$ or without $(-)$ transfection of MT-SENP2 isoforms as indicated. Overexpression of SENP2 (d), but not SENP2-M (f) or SENP2-S (h), inhibits the p53-mediated transcription. The p53-I- and p53 + I + HCT116 cells were co-transfected with p53-luc (a luciferase reporter under control of p53-response elements) and MT (-) or MT-SENP2 (+) DNA plasmids. RLA shows the activity of p53-dependent transcription $(n=3)$. Actin level is used as a loading control for immunoblot analysis. (i) Diagram illustrating the three isoforms of SENP2. NLS, nuclear localization sequence; NES, nuclear export signal

HCT116-SENP2S, cells, we detected a resistance to cell death induced by doxorubicin (Figure 2c, $n=3$ ). Immunostaining of activated caspase-3 and TUNEL staining analyses further indicated that the doxorubicin-induced apoptosis was 
a

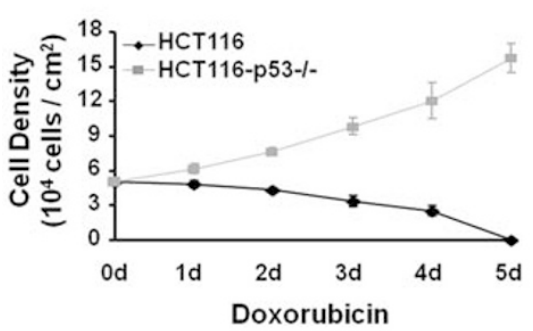

b

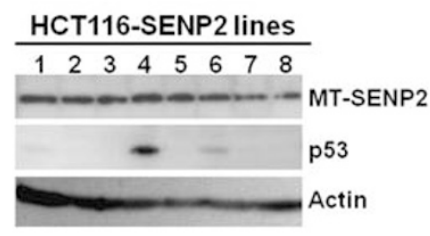

HCT116-SENP2M lines

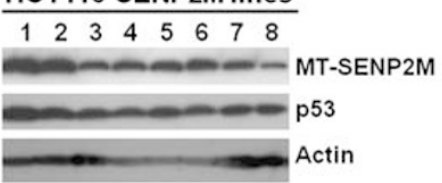

HCT116-SENP2S lines

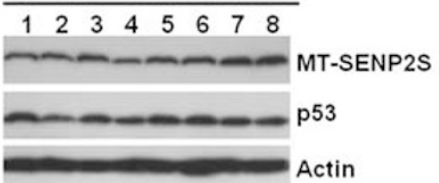

C

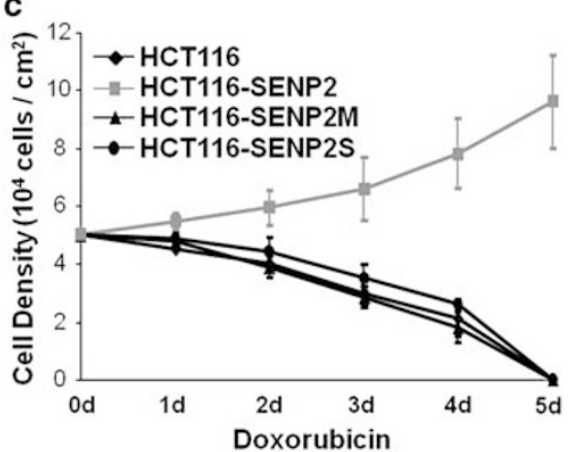

d
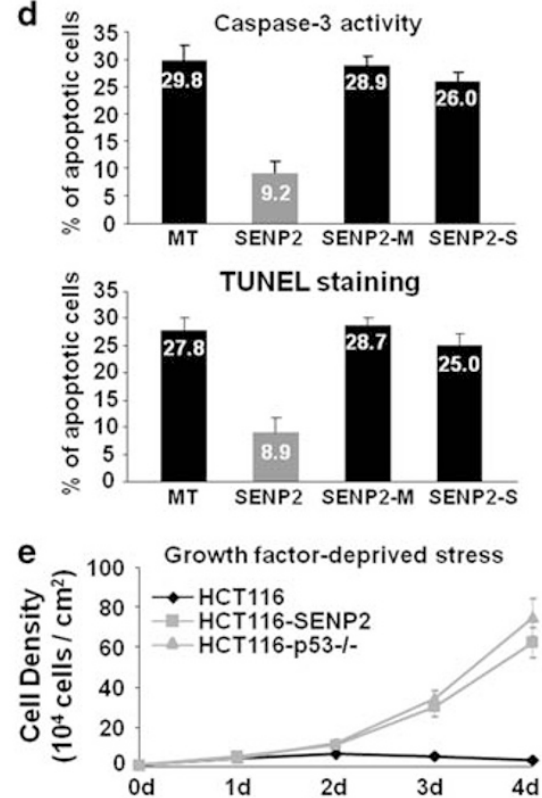

Figure 2 SENP2 interferes with the DNA damage-induced cell death and the growth factor-deprived stress mediated by p53. (a) Disruption of p53 results in the survival of HCT116 cells treated with doxorubicin. (b) Immunoblot analysis indicates the expression of SENP2 isoforms, SENP2, SENP2-M and SENP2-S, in HCT116 stably transformed variants, HCT116-SENP2, HCT116-SENP2M and HCT116-SENP2S, respectively. Repression of p53 is detected in majority of the HCT116-SENP2 sublines, but not the HCT116-SENP2M and HCT116-SENP2S derivatives. The number indicates the different stably transformed lines. Actin level is also analyzed as a loading control. (c) The doxorubicin-mediated cell survival problem is lost in the HCT116-SENP2, but not in HCT116-SENP2M and HCT116-SENP2S, cell lines. (d) Expression of the myc-tagged (MT) SENP2, but not SENP-M, SENP2-S or MT, has a preventive effect on the percentage of HCT116 cells undergoing DNA damage-induced apoptosis with doxorubicin treatment for $24 \mathrm{~h}$ as determined by immunostaining of activated caspase-3 and TUNEL staining. (e) HCT116-SENP2 variants expressing high levels of SENP2 are able to grow under a growth factor-deprived stress condition, reminiscent to the knockout of p53 in HCT116 cells

drastically diminished by high levels of SENP2, but not SENP2-M and SENP2-S (Figure 2d, $n=3$ ). The stress response induced by growth factor deprivation has also been shown to be dependent on p53. ${ }^{18}$ We therefore tested if high levels of SENP2 expressed in the HCT116-SENP2 cell lines caused an effect similar to the loss of p53. In agreement with prior reports, we found that HCT116 cells with p53 deficiency continue to grow under serum starvation (Figure $2 \mathrm{e}$ ). A similar growth curve was observed in the HCT116-SENP2 cells compared with the HCT116-p53-/- mutants (Figure 2e, $n=3$ independent cell lines). High levels of SENP2 resulted in growth of HCT116 cells under growth factor-deprived condition, normally leading to cell senescence. The isoform-specific regulation of SENP2 apparently had a role in the p53-dependent stress responses.
Differential localizations of the SENP2 isoforms. To elucidate the mechanism underlying the differential regulation of $\mathrm{p} 53$ by SENP2, we first investigated the subcellular distribution of different SENP2 isoforms. Similar results were obtained in all three different cell lines, HCT116, C57MG and $\mathrm{C} 3 \mathrm{H} 10 \mathrm{~T} 1 / 2$, examined. Immunostaining analysis revealed that the myc-tagged SENP2 is preferentially located to the nucleus, exhibiting the nuclear plasma (Figures $3 \mathrm{a}$ and $\mathrm{b}$ ) or dotted staining pattern (Figures $3 \mathrm{c}$ and d). The SENP2-M form displayed an accumulation in cytoplasmic vesicles and the perinuclear region (Figures $3 e$ and $f$ ), while the SENP2-S form distributed rather evenly in the cytoplasm (Figures $3 g$ and $h$ ). Double labeling with lamin B, a nuclear envelope protein, further indicated the predominant nuclear and cytoplasmic distributions of SENP2 

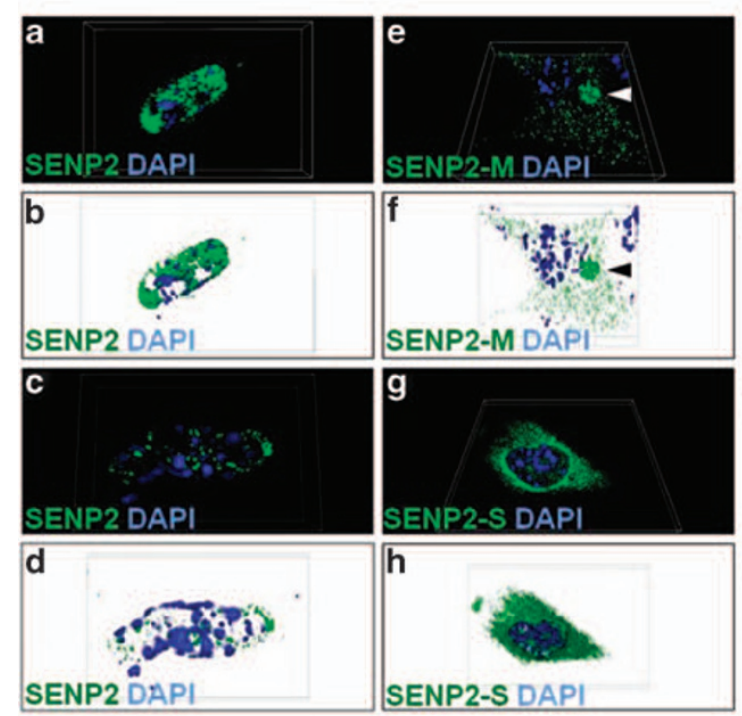
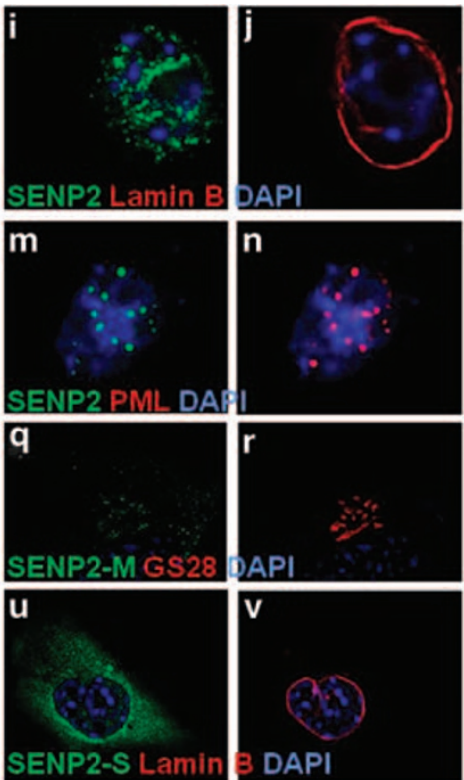
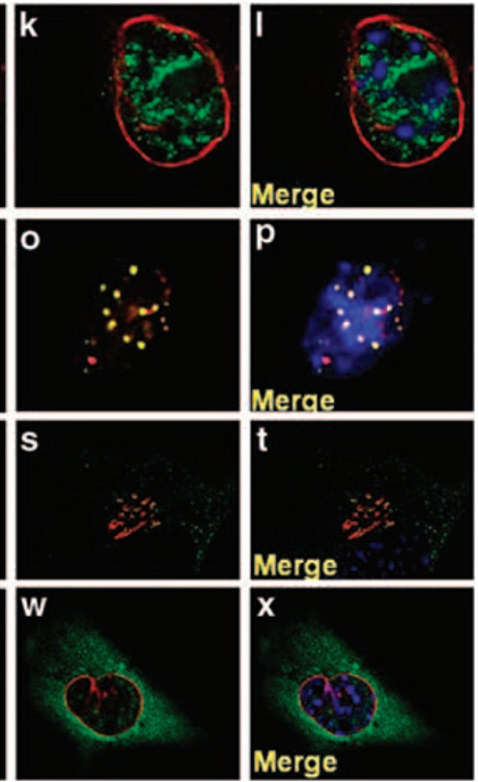

Figure 3 Differential localizations of SENP2 isoforms. Three different isoforms of the myc-tagged SENP2, SENP2 (a-d and i-p), SENP2-M (e, $\mathbf{f}$ and $\mathbf{q}-\mathbf{t}$ ) and SENP2-S $(\mathbf{g}, \mathbf{h}$ and $\mathbf{u}-\mathbf{x})$ are transiently expressed in C57MG mammary epithelial cells. 3D images (a, $\mathbf{c}, \mathbf{e}$ and $\mathbf{g})$ of immunostained cells as well their shadow views (b, $\mathbf{d}, \mathbf{f}$ and $\mathbf{h})$ reveal their subcellular distributions (green). Arrowheads indicate the perinuclear staining of SENP2-M (e and f). (i-x) Sectioned views show double labeling of SENP2 isoforms (green) with subcellular markers (red), lamin B (i-I and $\mathbf{u}-\mathbf{x})$, PML $(\mathbf{m}-\mathbf{p})$ and GS28 (q-t). Immunostained cells were counterstained by DAPI (blue). Three-color merge images $(\mathbf{l}, \mathbf{p}, \mathbf{t}$ and $\mathbf{x})$, SENP2 and DAPI (i, $\mathbf{m}, \mathbf{q}$ and $\mathbf{u})$, marker and DAPI (j, $\mathbf{n}, \mathbf{r}$ and $\mathbf{v})$; SENP2 and marker ( $\mathbf{k}, \mathbf{0}$, $\mathbf{s}$ and $\mathbf{w})$ show localization of SENP2 isoforms in the cell

(Figures 3i-l) and SENP2-S (Figures $3 u-x$ ), respectively. Occasionally, we found SENP2-S in both the nucleus and cytoplasm (data not shown) that might contribute to the slight reduction of p53 (Figure 1g). Co-localization of the Golgi marker GS28 with SENP2-M implied that it functions in the secretory pathway (Figures $3 q-t)$. PML co-localizes with the nuclear dotted staining of SENP2, suggesting that it accumulates in the PML bodies under certain circumstances (Figures $3 m-p)$. Many sumoylated proteins are targeted to these specific structures, ${ }^{4}$ including p53. ${ }^{19,20}$ As SUMO modification of PML and p53 is also a key determinant for maintaining genome integrity, ${ }^{4}$ this raises the possibility of SENP2 modulating the p53/Mdm2 circuit at the PML bodies.

Effects of SUMO modification on Mdm2 localization. We previously showed that SENP2 has a role in the sumoylation status of Mdm2 ${ }^{7}$ cellular compartmentalization of which may be regulated by the SUMO pathway. To determine the cellular trafficking of Mdm2 dictated by sumoylation, we examined the subcellular distribution of GFP-tagged Mdm2 and Mdm2-SUMO1 in three different cell lines: HCT116 (Figure 4), C57MG (data not shown) and C3H10T1/2 (data not shown). Although Mdm2 was mainly found in the nuclear plasma (Figures 4a-c), Mdm2-SUMO1 (Mdm2-SUMO) displayed nuclear dotted staining (Figures $4 d-f$ ). A similar nuclear dotted pattern was detected using Mdm2SUMO1 ${ }^{\mathrm{GG} 96-97 \Delta}\left(\mathrm{Mdm}^{-S U M O}{ }^{\Delta G G}\right.$ ), a mutant lacking the last two glycine residues of SUMO1 and preventing further conjugation that might affect subcellular distribution (Figures $4 \mathrm{~g}-\mathrm{i})$. Co-localization analysis further revealed that the SUMO-conjugated Mdm2 is located in the PML bodies
(Figures $4 \mathrm{j}-\mathrm{m}$ ). These results suggest that the localization of Mdm2 to the PML bodies may be modulated by the SUMO pathway.

Co-localization of SENP2 with SUMO-conjugated Mdm2. The accumulation of SENP2 (Figure 3) and Mdm2-SUMO1 (Figure 4) in the PML bodies suggested that these two molecules interact with each other. SENP2 might have a crucial role in SUMO-dependent regulation of $\mathrm{Mdm} 2$. We therefore tested whether SENP2 co-localizes with Mdm2 and whether SUMO conjugation alters this co-localization. Although SENP2 and Mdm2 showed similar patterns of nuclear distribution, their co-localization appeared to be minimal, with only few spots containing both proteins (Figures 5a-d). SENP2-M and SENP2-S also did not co-localize with Mdm2 (Figures $5 \mathrm{e}-1$ ). When the distribution of SENP2, Mdm2SUMO1 and PML was examined, we identified extremely high levels of co-localization between these molecules (Figures $5 \mathrm{~m}-\mathrm{p}$ ). To ensure that this redistribution of SENP2 is not due to a general association of SUMO proteases with SUMO1 polypeptides, we showed that the expression of Mdm2SUMO1 does not induce the accumulation of SENP2-M and SENP2-S in the PML bodies (Figures $5 q-x$ ). These findings, together with the data presented earlier (Figures 3 and 4), imply that SENP2 regulates desumoylation of Mdm2 at the PML bodies.

Association of SENP2 with Mdm2. The co-localization of SENP2 with SUMO-conjugated Mdm2 led us to examine their association. Co-immunoprecipitation was performed to identify formation of the SENP2-Mdm2 complex in HCT116 cells transiently expressing MT-SENP2 with GFP-tagged 

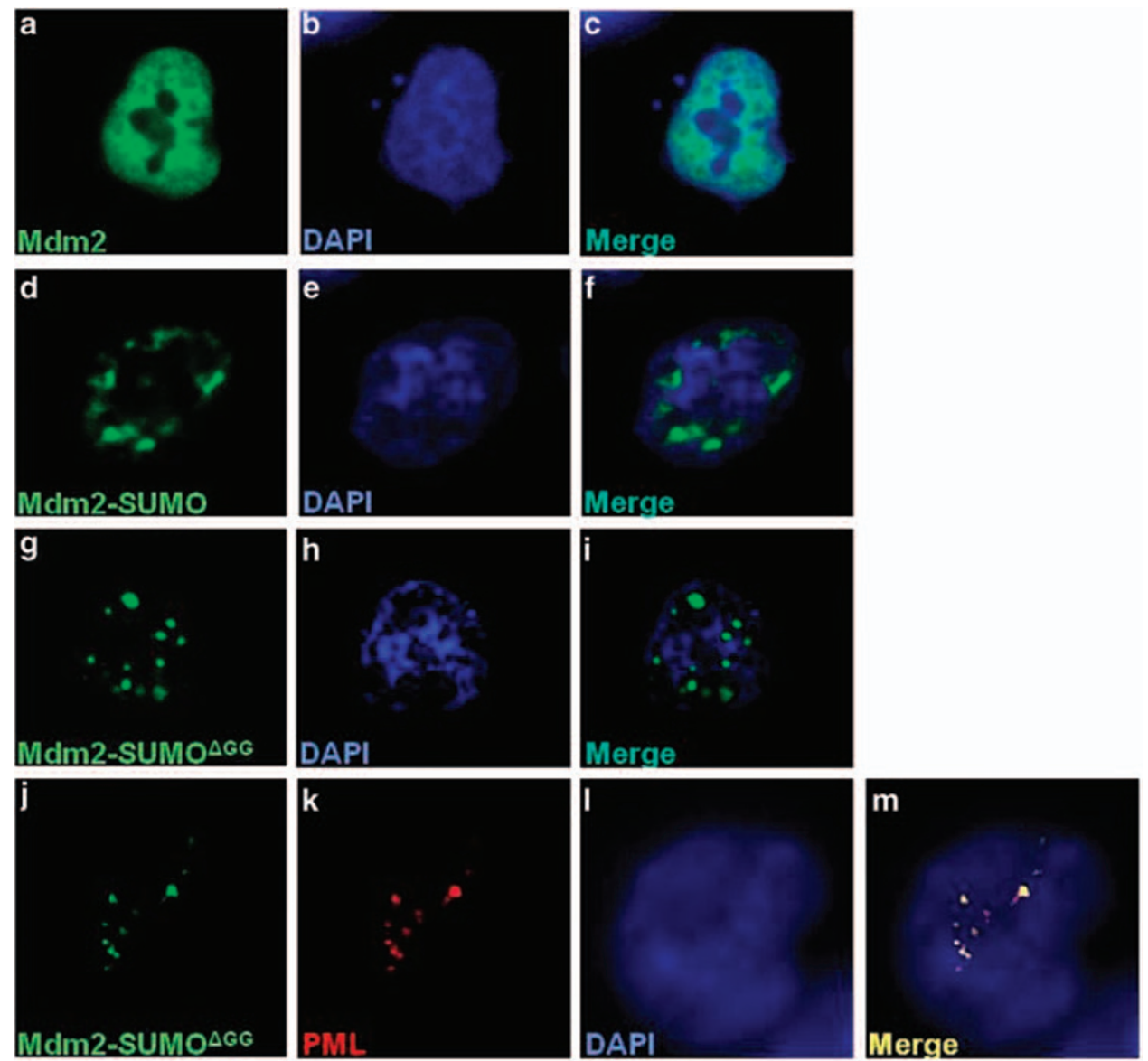

Figure 4 SUMO conjugation affects the subcellular distribution of Mdm2. HCT116 cells transfected with GFP-tagged Mdm2 (a-c), Mdm2-SUMO (d-f) or Mdm2$\mathrm{SUMO}^{\Delta \mathrm{GG}}(\mathbf{g}-\mathbf{m})$, were analyzed by fluorescent imaging. $(j-\mathrm{m})$ Co-localization analysis reveals that the nuclear dotted staining of Mdm2-SUMO ${ }^{\Delta \mathrm{GG}} \mathrm{Co}-\mathrm{localizes}$ with $P M L$ in HCT116 cells

Mdm2 or Mdm2-SUMO1. Indeed, SENP2 was identified in complexes containing either Mdm2 or Mdm2-SUMO1 (Figure 6a). It seems that SUMO conjugation of Mdm2 facilitates the complex formation between these two molecules. Similar observation was also found in HCT116SENP2 cells where SENP2 expression is elevated (Figure 6b). Together with the co-localization study, these data suggest that the interaction of SENP2 and Mdm2 is regulated by the SUMO pathway at the PML bodies.

SENP2 catalyzes the desumoylation process of Mdm2. We previously found that SUMO-conjugated Mdm2 is elevated in SENP2-null cells. ${ }^{7}$ Reintroducing SENP2 was able to diminish the sumoylated Mdm2 in the mutants. ${ }^{7}$ We therefore initiated a biochemical approach to further determine the effect of SENP2 on the desumoylation of Mdm2. First, we investigated if overexpression of SENP2 alters the sumoylation status of endogenous Mdm2 in cells with either transient or stable expression of SENP2 at high levels. The result showed that transient expression of SENP2 reduces the sumoylated Mdm2, while the Mdm2 level remains comparable (Figure 7a). Similar results were obtained by comparing the sumoylated Mdm2 levels in HCT116 and HCT116-SENP2 cells (Figure $7 b$ ). Second, we used a cell free system to determine if high levels of SENP2 present in the protein extract can affect the SUMO-conjugated Mdm2 level. MT or MT-SENP2 protein, purified by immunoprecipitation, was incubated with protein extracts of cells expressing high levels of SUMO1 in vitro. The sumoylated levels of endogenous Mdm2 were then analyzed by immunoblot analysis. In the extracts treated with MT-SENP2, the level of SUMO-conjugated Mdm2 significantly reduced (Figure 7c). This conversion of Mdm2 was prevented by the addition of $\mathrm{N}$-ethylmaleimide (NEM), a SUMO protease inhibitor, suggesting that the effect is specific to the SUMO protease activity of SENP2 (Figure 7c). Finally, we examined the ability of SENP2 to remove SUMO from Mdm2 using in vitro reconstitution analysis (Figure 7d). Recombinant enzymes were first utilized to perform SUMO conjugation of Mdm2. The addition of purified SENP2 efficiently revered the sumoylation process of Mdm2, suggesting that it is an SENP2 substrate.

SENP2-dependent regulation is sensitive to the p53-binding activity of Mdm2. To decipher the regulatory pathway underlying the modulation of p53 by SENP2 and Mdm2, we first examined whether Mdm2 has an essential role in this process. As expected, the knockdown of Mdm2 by RNA interference increased the levels of p53 and its downstream 

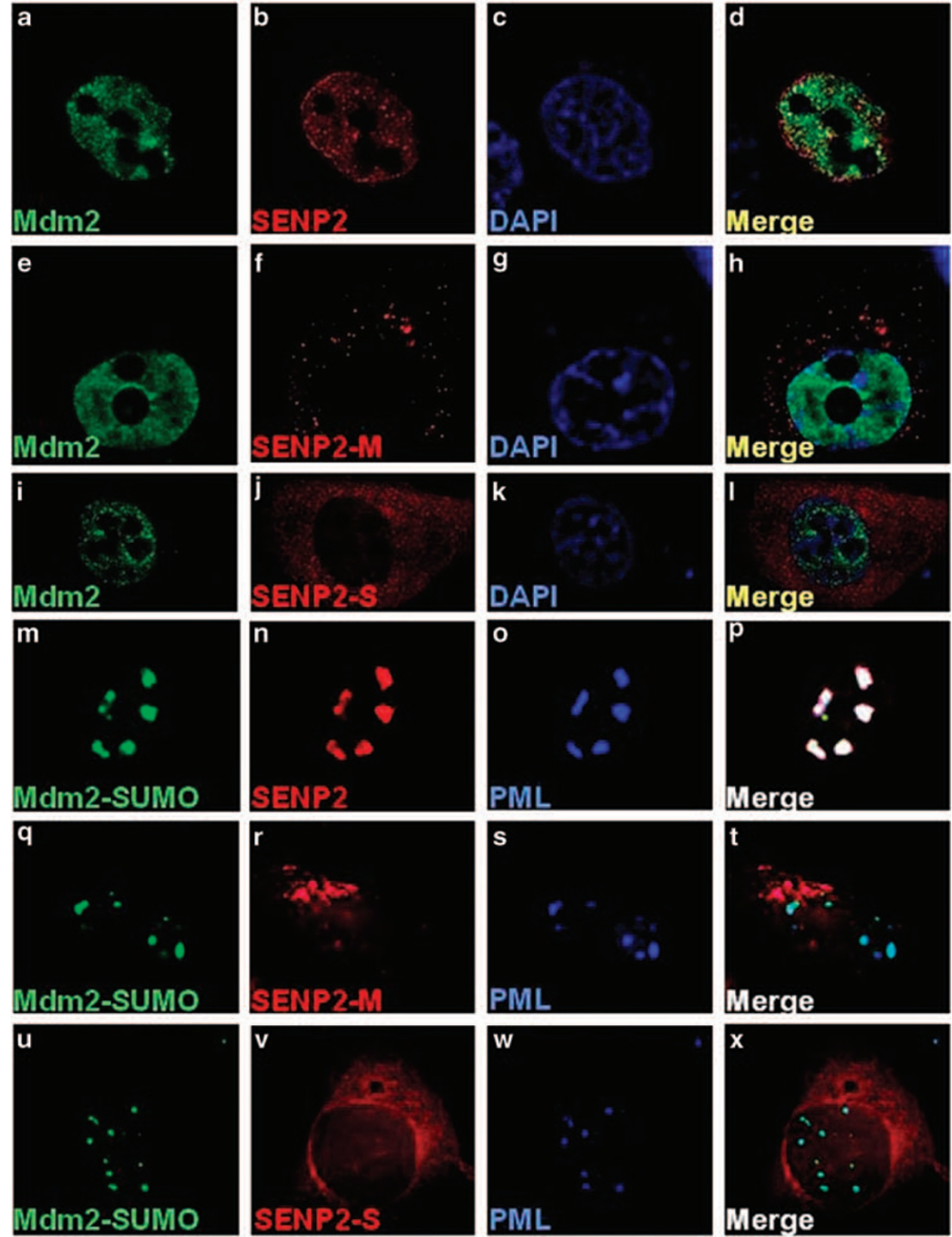

Figure 5 Isoform-specific co-localization of SENP2 with SUMO-conjugated Mdm2. HCT116 cells transfected with GFP-tagged Mdm2 (a-I) or Mdm2-SUMO ( $\mathbf{m}-\mathbf{x})$, and different isoforms of myc-tagged SENP2, were analyzed by fluorescent imaging. (a-l) Double-labeling analysis shows no co-localization of Mdm2 with SENP2 (a-d), SENP2$\mathrm{M}(\mathbf{e}-\mathbf{h})$ and SENP2-S (i-l). $(\mathbf{m}-\mathbf{p})$ Triple labeling of cells with Mdm2-SUMO, PML and SENP2 isoforms reveals that SENP2 ( $\mathbf{m}-\mathbf{p})$, but not SENP2-M (q-t) and SENP2-S $(\mathbf{u}-\mathbf{x})$, co-localizes with SUMO-conjugated Mdm2 at the PML bodies

target p21 (Figure 8a). High levels of SENP2 reduced the levels of sumoylated Mdm2, p53 and p21 (Figure 8a). However, when Mdm2 was knocked down, high levels of SENP2 were no longer capable of reducing the levels of p53 and p21, suggesting a requirement of Mdm2 in the regulation of p53 mediated by SENP2 (Figure 8a). Next, we determined if the SENP2-mediated effect of Mdm2 is dependent or independent of the Mdm2 and p53 binding. Nutlin-3 is a potent small-molecule antagonist, which binds to the p53-binding pocket of Mdm2 and prevents their interaction, thereby stabilizing p53 and increasing the level of p21
(Figure 8b). We found that the SENP2-mediated downregulation of p53 and p21, but not SENP2-mediated desumoylation of Mdm2, is sensitive to Nutlin-3 treatment (Figure 8b), suggesting that SENP2 acts upstream of the Mdm2 and p53 interaction. We then examined if SUMO conjugation has an effect on Mdm2-dependent p53 degradation. A reduction of p53 occurred when Mdm2 was expressed (Figure 8c). However, expression of Mdm2SUMO1 or Mdm2-SUMO ${ }^{\Delta G G}$ did not cause a reduction, but a slight increase in p53 level (Figure 8c). Furthermore, presence of Nutlin-3 not only abolished the reduction of p53 
a

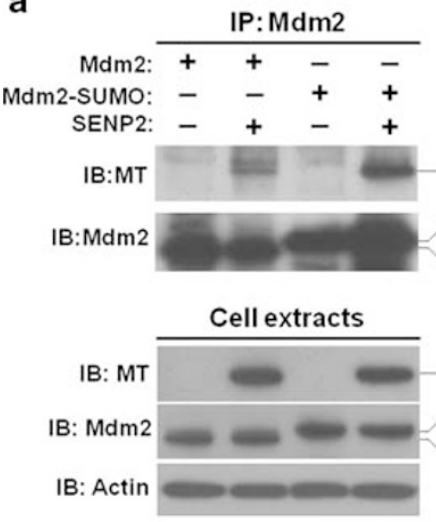

b

SENP2

Mdm2-SUMO

Mdm2

SENP2

Mdm2-SUMO

$\mathrm{Mdm} 2$

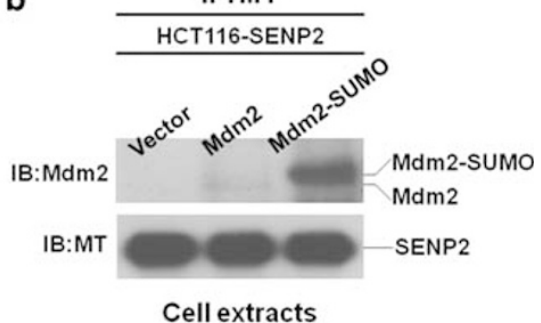

IB: MT

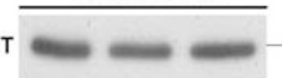

SENP2

IB: Mdm2

IB: Actin
Mdm2-SUMO

Mdm2

Figure 6 SENP2 associates with Mdm2 and Mdm2-SUMO. Co-immunoprecipitation reveals that SENP2 forms protein complexes with Mdm2 and Mdm2-SUMO. (a) HCT116 cells, which transiently express either Mdm2 or Mdm2-SUMO with or without MT-SENP2, were analyzed by immunoprecipitation with anti-Mdm2 antibodies, followed by immunoblot with anti-MT or anti-Mdm2 antibodies. (b) HCT116-SENP2 cells, which were transfected by GFP-tagged Mdm2 or Mdm2-SUMO, were analyzed by immunoprecipitation with anti-MT antibodies, followed by immunoblot with anti-Mdm2 or anti-MT antibodies. Total cell extracts directly analyzed by immunoblot analysis show the protein expression levels ( $\mathbf{a}$ and $\mathbf{b}$ ). Actin level is used as a loading control

a
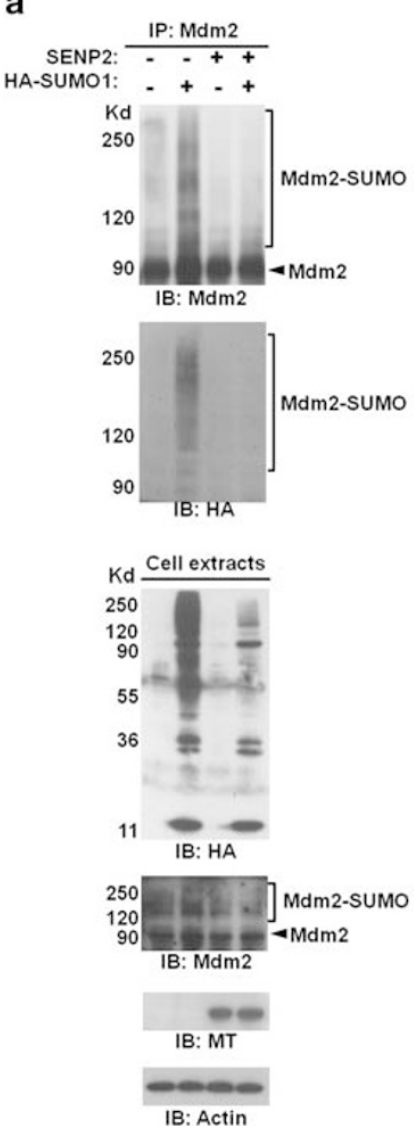

b
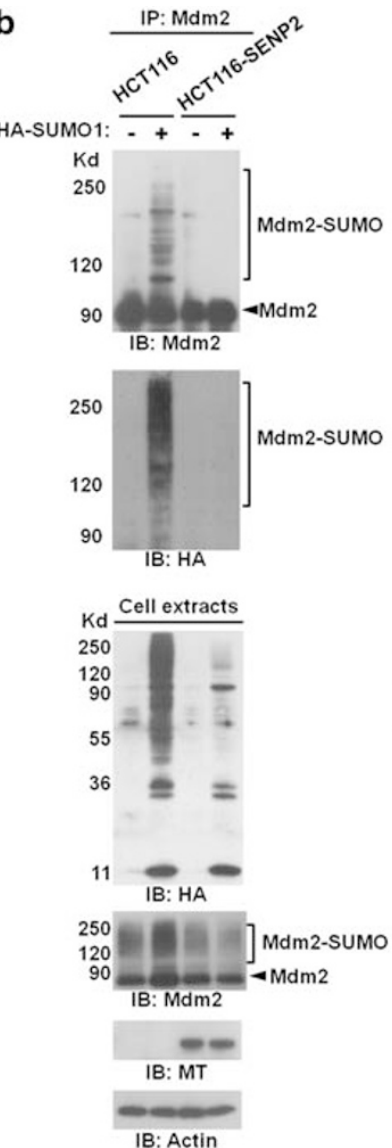

c

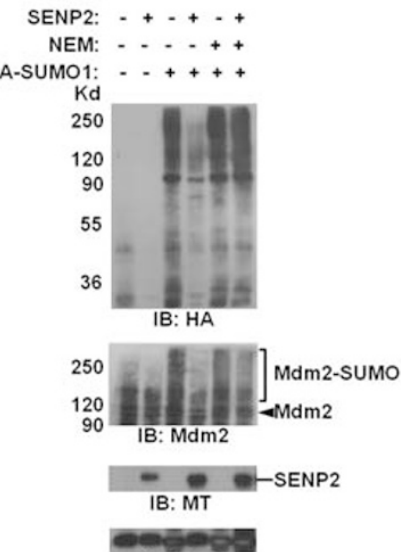

IB: Actin

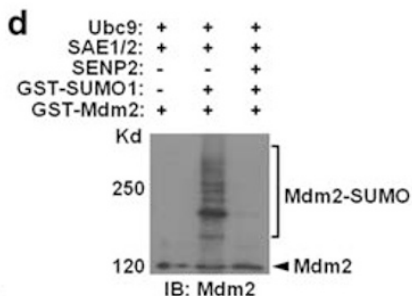

IB: Mdm2

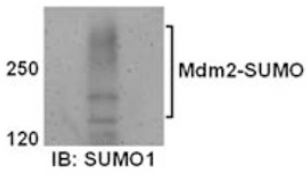

Figure 7 SENP2 regulates the SUMO modification of Mdm2. SENP2-mediated desumoylation of Mdm2 is analyzed by the in vivo (a and $\mathbf{b})$ and in vitro (c and $\mathbf{d})$ systems. $(\mathbf{a}$ and $\mathbf{b}$ ) The effect of SENP2 expression on the sumoylation status of endogenous Mdm2 is assayed by IP-IB analysis. The SENP2 expression reduces the sumoylated Mdm2 in the HA-SUMO1 transfected HCT116 cells (a) and HCT116-SENP2 cells (b). Total cell extracts directly analyzed by immunoblot analysis show the protein expression levels. Actin level is used as a loading control. (c) In vitro desumoylation assay shows that the addition of purified MT-SENP2 (+), but not MT (-), diminishes the level of SUMO-conjugated Mdm2 while the presence of SUMO protease inhibitor, NEM, prevents the desumoylation activity. Protein extracts, isolated from the HA-SUMO1transfected HCT116 cells, were treated with purified SENP2 or MT, with or without the presence of NEM, followed by immunoblot analyses. (d) In vitro reconstitution analysis using recombinant enzymes and substrates reveals that SUMO conjugation of Mdm2 is reversed by purified SENP2. GST-tagged Mdm2 was sumoylated by recombinant Ubc9, SAE1/2 and GST-SUMO1 proteins, followed by desumoylation with purified SENP2 
a

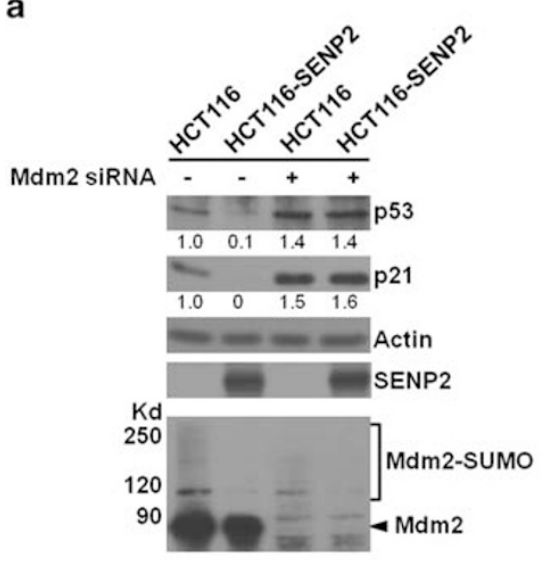

C

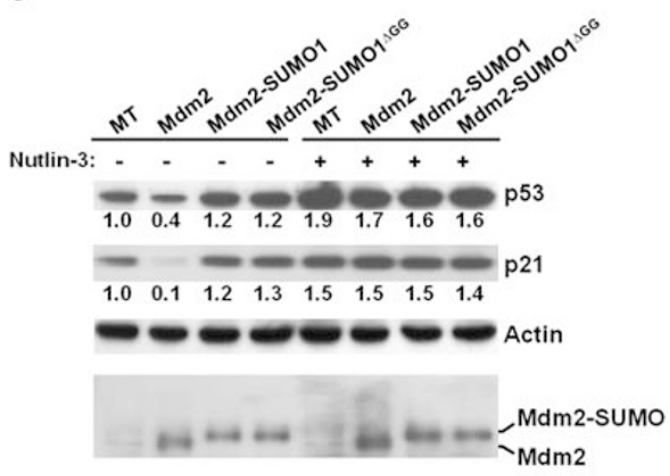

b

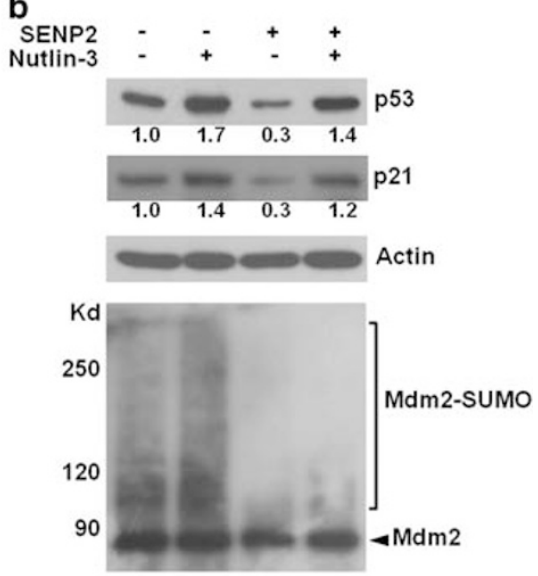

d

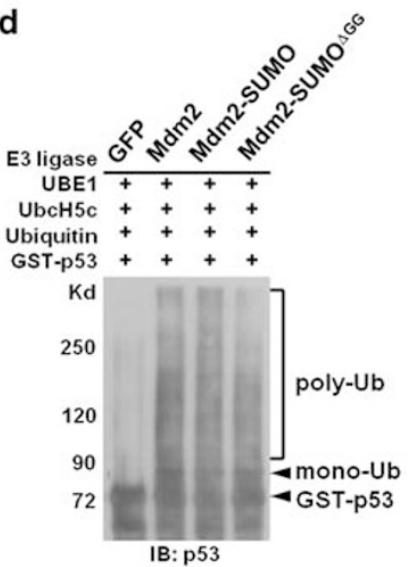

Figure 8 SENP2-dependent SUMO modification of Mdm2 modulates the p53 level. (a) Mdm2 is required for SENP2-dependent regulation of p53. Immunoblot analysis shows the levels of p53 and p21 affected by Mdm2 RNA interference (Mdm2 siRNA) in HCT116 and HCT116-SENP2 cells. (b) SENP2-mediated repression of p53 is sensitive to the inhibition of Mdm2 by Nutlin-3. Immunoblot analysis shows the levels of p53 and p21 affected by the SENP2 expression in HCT116 cells in the presence or absence of Nutlin-3. The expression of SENP2 also reduces the level of Mdm2-SUMO independent of Nutlin-3. (c) Effects of Nutlin-3 on the sumoylation of Mdm2 and Mdm2-mediated regulation of p53 and p21. Immunoblot analysis reveals that Nutlin-3 interferes with the p53 and p21 levels by the expression of MT, Mdm2, Mdm2-SUMO and Mdm2$\mathrm{SUMO}^{\triangle \mathrm{GG}}$ in HCT116 cells. (d) The ubiquitin E3 ligase activity is maintained in the fusion proteins of Mdm2 and SUMO1. In vitro ubiquitination assay reveals that purified GFP-tagged Mdm2, Mdm2-SUMO and Mdm2-SUMO ${ }^{\Delta G G}$, but not GFP, are able to modify a recombinant GST-tagged p53 protein with ubiquitin. Actin level is used as a loading control for immunoblot analysis

caused by Mdm2, but also significantly elevated the p53 level (Figure 8c). Even in the presence of Mdm2-SUMO1 and Mdm2-SUMO ${ }^{\Delta G G}$, we observed a consistent elevation of p53 upon Nutlin-3 treatment (Figure 8c). To ensure that the observed effects are not due to a disruption of the E3 ligase activity, we examined the ability of the Mdm2 fusion proteins to modify p53. In an in vitro ubiquitination assay, purified GFP-tagged Mdm2, Mdm2-SUMO and Mdm2-SUMO ${ }^{\Delta G G}$, but not GFP, were capable of conjugating a recombinant GST-tagged p53 protein (Figure 8d), suggesting that the E3 ligase activity is maintained in the fusion proteins. Our findings imply that the SENP2-dependent modification of Mdm2 by the SUMO pathway acts upstream of the binding of Mdm2 and p53. The binding inhibition mediated by Nutlin-3 resulted in insensitiveness of Mdm2 to the SUMO regulation.

SUMO regulation of $\mathrm{Mdm} 2$ in DNA damage-induced apoptosis. The ability of SENP2 to modify Mdm2 and the accumulation of SENP2 and sumoylated Mdm2 in the PML bodies led us to investigate the differential localizations possibly associated with p53-mediated cellular responses. We first examined whether the subcellular distribution of Mdm2 is altered in DNA damage-induced apoptosis. In HCT116 cells undergoing p53-dependent apoptosis upon doxorubicin treatment, we found accumulations of Mdm2 (Figure 9a), but not p53 (Figure 9b), in the PML bodies. None of them showed PML body staining of Mdm2 before doxorubicin treatment or after doxorubicin addition for $2 \mathrm{~h}$ (Figure 9c). However, the increasing number of cells exhibiting the PML body staining of Mdm2 (8h: $7.8 \%, 24 \mathrm{~h}$ : $20.6 \%$ and $36 \mathrm{~h}: 26.5 \%)$ coincides with those undergoing apoptosis ( $8 \mathrm{~h}: 10.3 \%, 24 \mathrm{~h}: 24.7 \%$ and $36 \mathrm{~h}: 34.1 \%$ ) during the course of doxorubicin treatment (Figure 9c). These phenotypes were accompanied by enhanced accumulations of SENP2 in the PML bodies (Figure 9d). In HCT116-SENP2 cells, the PML body distribution of Mdm2 and doxorubicininduced apoptosis were prevented by high levels of SENP2, suggesting that it is involved in these regulatory processes (Figure 9c). Upon doxorubicin treatment, sumoylation of Mdm2 is enhanced, which is accompanied by elevated levels 

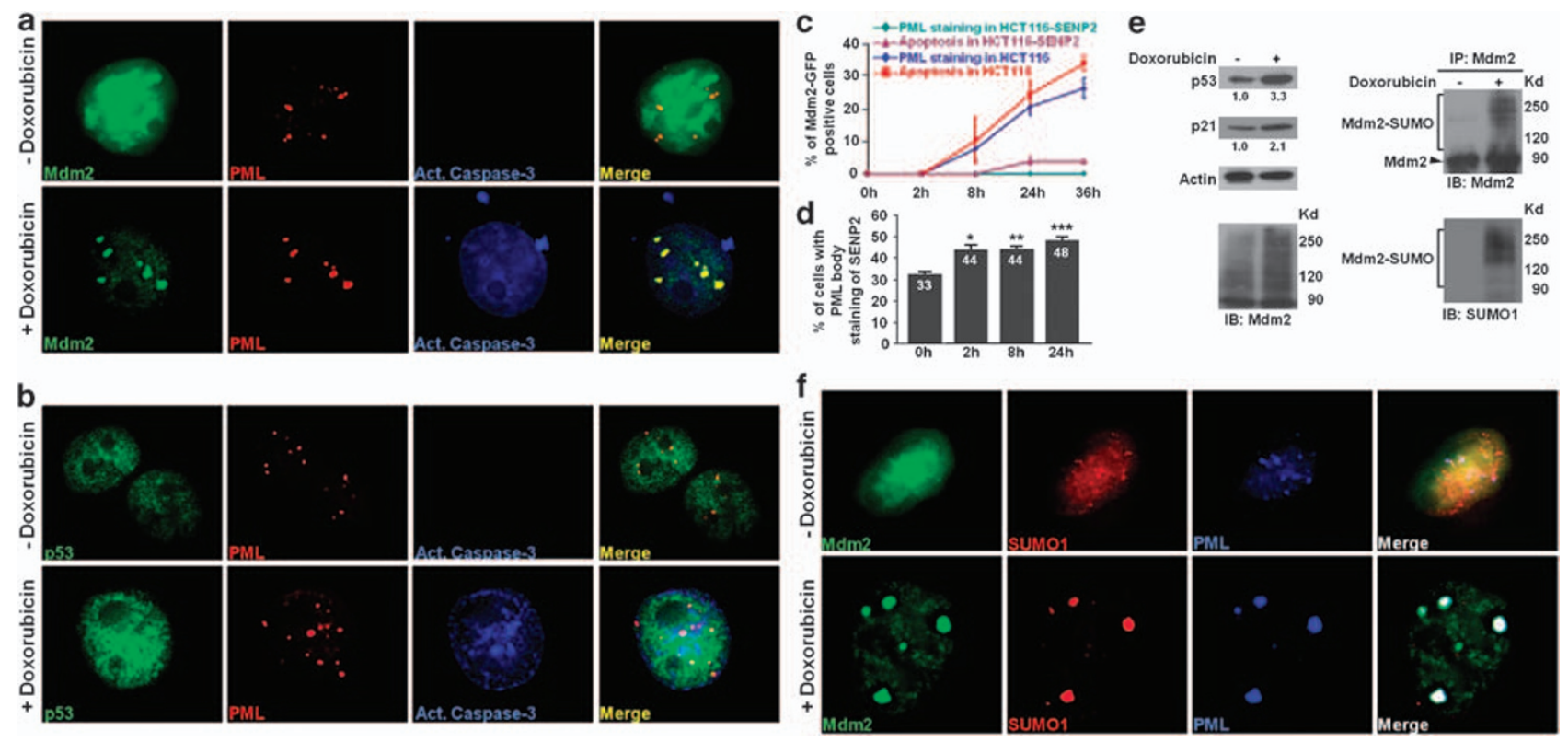

Figure 9 DNA damage-induced apoptosis promotes the interaction of Mdm2 and SENP2 at the PML bodies. (a) Triple labeling of Mdm2, PML and activated caspase-3 reveals that Mdm2 accumulates in the PML bodies of cells undergoing p53-dependent apoptosis. Mdm2-GFP was expressed in HCT116 cells without or with the addition of doxorubicin for $24 \mathrm{~h}$. Mdm2 co-localized with PML in cells undergoing apoptosis identified by immunostaining of activated caspase-3. (b) Triple labeling of p53, PML and activated caspase-3 shows that the doxorubicin-induced apoptosis does not promote p53 accumulation in the PML bodies. (c) Graph indicating the percentage of Mdm2-GFP positive cells displaying PML body distribution, and undergoing programmed cell death in the course of doxorubicin treatment. (d) Graph showing the percentage of HCT116SENP2 cells displaying the PML body distribution of SENP2 in the course of doxorubicin treatment $\left(P\right.$-value: $\left.{ }^{*} P<0.035 ;{ }^{* \star} P<0.021 ;{ }^{* * *} P<0.017\right)$. (e) Immunoblot and immunoprecipitation-immunoblot analyses reveal that the induction of p53 and p21 coincides with the accumulation of sumoylated Mdm2 upon doxorubicin treatment. Actin level is used as a loading control. (f) DNA damage response promotes accumulations of Mdm2 and SUMO1 in the PML bodies. HCT116 cells transfected with GFP-tagged $\mathrm{Mdm} 2$ were cultured in the presence or absence of doxorubicin for $24 \mathrm{~h}$, followed by triple labeling analysis

of p53 and p21 (Figure 9e). Before the addition of doxorubicin, free SUMO1 as well as Mdm2 proteins did not localize to the PML bodies (Figure 9f). However, doxorubicin promoted the accumulations of Mdm2 and SUMO1 to the PML bodies (Figure 9f). This differential co-localization implies that sumoylated Mdm2 accumulates in the PML bodies in DNA damage-induced responses. The data suggest that the SUMOdependent regulation of $\mathrm{Mdm} 2$ distribution may have a crucial role in genotoxic stresses mediated by $\mathrm{p} 53$.

\section{Discussion}

This study demonstrated an isoform-specific effect of SENP2 on the p53/Mdm2 regulatory circuit. This effect is likely attributed to the differential compartmentalization of SENP2 isoforms within the cells. The longest form of SENP2 is necessary and sufficient for modulating the stability of p53, and the p53-dependent transcription and stress responses. Biochemical studies further show that SENP2 promotes the desumoylation of Mdm2, contributing to the p53-dependent regulations. The accumulation of SENP2 in the PML bodies further indicates its importance in modifying the activity of Mdm2 and p53. Although both SENP2 and Mdm2 preferentially accumulate in the nucleus, their localizations do not seem to overlap significantly. However, SUMO conjugation of Mdm2 leads to protein translocation, resulting in strong accumulations in the PML bodies where it co-localized significantly with SENP2. The PML body distribution of Mdm2 and SENP2 is also modulated by doxorubicin-induced
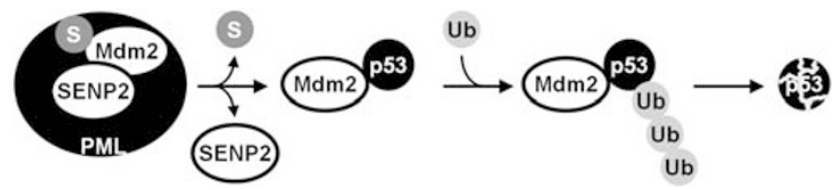

Figure 10 Model for SENP2-mediated modulation of Mdm2 in regulating p53. The diagram illustrates the mechanism underlying the regulation of Mdm2 by the SUMO pathway to control the cellular level of p53. SENP2 regulates p53 through modulation of Mdm2. SENP2 interacts with sumoylated Mdm2 and regulates its SUMO conjugation at the PML body. The SENP2-dependent SUMO modification acts upstream of the binding of Mdm2 and p53 in the regulatory pathway. Desumoylation of $\mathrm{Mdm} 2$ permits its binding and ubiquitination of $\mathrm{p53}$, which is then degraded through the proteolysis system

apoptosis, suggesting a role in stress responses mediated by p53. On the basis of this work, we have proposed a mechanism for the SENP2-Mdm2-p53 regulatory pathway (Figure 10). Although p53 does not seem to be regulated by SENP2 at the PML bodies, it is possible that SENP2 may also catalyze the desumoylation of p53. If true, this would add complexities to this multilayered regulatory network. However, the SENP2-mediated SUMO modification of p53 might have functional consequences distinct to that of Mdm2, which remains to be explored.

We have previously reported that Nutlin-3 treatment is still capable of increasing the p53 level in SENP2-null cells. ${ }^{7}$ Nutlin-3 elevates p53 under normal (SENP2 $+/+$ ) and hypersumoylated (SENP2-/-) conditions of Mdm2, implying that sumoylated Mdm2 is still able to interact with p53. Similar to 
that observed in SENP2-null cells, Nutlin-3 treatment on Mdm2-SUMO1 causes an elevation effect of p53. One possibility is that both Mdm2 and sumoylated Mdm2 form complexes with p53. Desumoylation of Mdm2 then activates its E3 ligase activity. The fact that E3 ligase activity is maintained in the Mdm2-SUMO fusion protein seems to argue against this. Alternatively, desumoylation of Mdm2 promotes the cytoplasmic translocation of p53 for degradation. Mdm2-SUMO and p53 could form a sequestration complex accumulating in the nucleus without initiating the degradation and nuclear-cytoplasmic translocation processes. Stabilization of p53 then is capable of promoting the subsequent responses to cellular stresses. The findings support our hypothesis, further implying that Mdm2-SUMO is sequestered at the PML bodies unable to promote p53 degradation under hyper-sumoylated conditions (Figure 10). Indeed, we have found that the cellular levels of sumoylated Mdm2 are increased upon doxorubicin treatment. Furthermore, Mdm2 and SUMO1 co-localize at the PML bodies of the doxorubicin-treated cells. Our data suggest that the SUMOconjugated Mdm2 recruits to the PML bodies, causing deficiency in targeting p53 degradation. However, due to the lack of information on identification of the sumoylation site of Mdm2, this hypothesis cannot be definitively assessed. Once the sumoylation site has been identified, it would be important to determine whether mutation of the sumoylation site interferes with the PML body distribution of Mdm2.

An important function of p53 as a guardian of the genome ensures that the genetic information is properly propagated during these processes. ${ }^{21}$ In addition, p53 preserves genomic stability in stress responses of various insults, including DNA damage, hypoxia, metabolic stress and oncogene activation. $^{22-24}$ The most documented mechanism by which p53 exerts its protective effects is as a transcription factor. ${ }^{25}$ SENP2 is apparently a negative regulator for the p53 level and the p53-dependent transcription. Although recent evidence has challenged the classical model of p53 activation, ${ }^{26}$ its stabilization remains to be a key regulatory step. SENP2, involved in the p53-induced regulations, may have a role in genotoxic stresses. Further investigation focusing on the action of SENP2 promises new insights into the mechanism underlying cellular stress mediated by the SUMO pathway. As our prior study indicated that the SENP2-Mdm2-p53 pathway is critical for the G-S checkpoint of mitotic divisions and endoreduplication, ${ }^{7}$ it is possible that aberrant stimulation of SENP2 causes cell cycle defects, leading to the prevention of cell death and senescence. Recent reports suggest that the SUMO pathway is involved in cellular responses to DNA double-strand breaks. ${ }^{27,28}$ Whether SENP2 participates in these genotoxic stresses essential for maintaining genome integrity remains an interesting question to be determined.

Mutations in the p53 gene have been linked to familial and sporadic forms of human cancer. Li-Fraumeni syndrome is a rare familial disorder that greatly increases the risk of developing several types of cancers in children and young adults. ${ }^{29}$ In sporadic forms, mutations have been identified in other key regulators, for example, p53, Mdm2 and p14 ${ }^{\mathrm{ARF}}$, which result in alteration of the p53 activity. ${ }^{22}$ Mice with disruption of RanBP2, a SUMO E3 ligase, developed tumors, ${ }^{30}$ suggesting that aberrant regulation of the SUMO pathway induces cells to undergo malignant transformation. Previous reports also suggest that SENP1 might be associated with development of prostate cancers. ${ }^{31}$ As SUMO can regulate p53 directly ${ }^{10}$ or indirectly, ${ }^{7}$ SENPs might contribute to the oncogenic processes at multiple levels. Therefore, it is conceivable that SENP2 has a crucial role in p53-induced tumorigenesis, which remains to be explored. However, embryonic lethality associated with the SENP2 deletion in mice prevents investigation of its oncogenic role. ${ }^{7}$ Mouse models permitting conditional inactivation of SENP2 are necessary to further decipher this genetic regulatory network.

\section{Materials and Methods}

DNA. The generation of pCS2-SENP2, pGFP-Mdm2, pGFP-Mdm2-SUMO1, pGEX-4T-SAE1/2 and pGEX-2T-Ubc9 clones was described previously. ${ }^{7,32,33}$ The pGFP-Mdm2-SUMO1 ${ }^{\text {GG96-97 }}$ clone was generated by mutating two glycine residues at the $C$ terminus of SUMO1 contained in the pGFP-Mdm2-SUMO1 plasmid using site-directed mutagenesis (Stratagene, La Jolla, CA, USA). To generate pGEX-2T-SUMO1, pGEX-2T-Mdm2 and pGEX-2T-p53 clones, the corresponding DNA fragments were inserted into pGEX-2T vector (GE HealthCare, Waukesha, WI, USA). The myc-tagged SENP2 cDNAs, encoding the middle and short isoforms, were inserted into the pCS2 vector to create the pCS2-SENP2-M and pCS2-SENP2-S clones, respectively. To generate the pHASUMO1 clone, SUMO1 fragment was inserted into the pCl-Neo-HA vector (Promega, Madison, WI, USA).

Cells. The establishment and culture of SENP2 $+/+$ and SENP2-/ - TS cell lines were described previously. ${ }^{7}$ To create the HCT116-SENP2, HCT116SENP2M and HCT116-SENP2S clones, DNA fragment containing pGK-Neo was first inserted into the pCS2-SENP2, pCS2-SENP2-M and pCS2-SENP2-S plasmids. The plasmids were then stably transfected into HCT116 cells, followed by $\mathrm{G} 418(800 \mu \mathrm{g} / \mathrm{ml})$ selection for 14 days. Colonies, which grow under drug resistance condition, were picked to establish cell clones. For DNA damage induction, cells were cultured with $1 \mu \mathrm{M}$ doxorubicin. For growth factor deprivation, cells were cultured in $0.1 \%$ fetal bovine serum. The number of cells was counted each day to assess the growth curve. Cells undergoing apoptosis were detected by immunostaining of activated caspase-3 or TUNEL staining (Millipore, Billerica, MA, USA) after the doxorubicin treatment for $24 \mathrm{~h}$. The stained images were taken randomly to determine the percentage of apoptotic cells by counting the activated caspase-3 or TUNEL-positive and -negative cells. Nutlin-3 $(10 \mu \mathrm{M})$ was added to the culture media to prevent the binding of p53 and Mdm2. To measure the p53dependent transcriptional activity, cells transfected with p53-luciferase, containing the luciferase reporter under control of p53 responsive elements, were subject to luciferase assay.

Immunostaining and immunoblot. Cells were fixed and subjected to immunological staining and fluorescent imaging analysis as described. ${ }^{34-38}$ Images were taken using Zeiss Axio Observer microscope, followed by deconvolution, 3D imaging and cut-view analyses. ${ }^{34,39}$ Immunoblot analysis was performed by isolating protein extracts from cells in the presence of protease inhibitor cocktail (Sigma-Aldrich, St. Louis, MO, USA), $1 \mathrm{mM}$ sodium molybdate, $1 \mathrm{mM}$ sodium vanadate and $1 \mathrm{mM}$ PMSF, or SDS lysis buffer (2\% SDS, $2 \%$ 2-mercaptoethanol, $10 \%$ glycerol, and $50 \mathrm{mM}$ Tris, $\mathrm{pH}$ 6.8), followed by electrophoresis as described. ${ }^{7,34,36,39,40}$ Bound primary antibodies were detected with horseradish peroxidase-conjugated secondary antibodies (Vector Laboratories, Burlingame, CA, USA), followed by ECL-mediated visualization (GE HealthCare) and autoradiography. ${ }^{7,34,36,39,40}$ Mouse monoclonal antibodies Actin (Thermo Fisher, Waltham, MA, USA), GS28 (BD Biosciences, San Jose, CA, USA), HA (Cell Signaling, Danvers, MA, USA), PML (Santa Cruz, Santa Cruz, CA, USA), Mdm2 (Santa Cruz), myc tag (Santa Cruz), p53 (Santa Cruz), SUMO1 (Invitrogen, Carlsbad, CA, USA), rabbit monoclonal antibody SUMO1 (Cell Signaling), rabbit polyclonal antibody caspase-3 (BD Biosciences), goat polyclonal antibodies lamin B (Santa Cruz) and p53 (Santa Cruz) were used in the analyses.

Ubiquitin, sumoylation and desumoylation assays. For in vitro ubiquitin assay, purified GFP-tagged Mdm2, Mdm2-SUMO1 or GFP-Mdm2$\mathrm{SUMO1}^{\triangle \mathrm{GG}}$ was added to the reaction buffer containing $50 \mathrm{mM}$ Hepes $(\mathrm{pH} 8.0)$, 
$1 \mathrm{mM}$ Mg-ATP, UBE1 (Boston Biochem, Cambridge, MA, USA), UbcH5c (Boston Biochem) and recombinant GST-p53 in a final volume of $10 \mu \mathrm{l}$, followed by incubation for $60 \mathrm{~min}$ at $37^{\circ} \mathrm{C}$. The reaction was then stopped by the addition of a loading buffer containing $150 \mathrm{mM}$ Tris- $\mathrm{HCl}(\mathrm{pH} 6.8), 6 \% \mathrm{SDS}, 30 \%$ glycerol and $6 \%$ 2-mercaptoethanol and analyzed by SDS-PAGE and immunoblot analysis of p53.

To perform the in vitro sumoylation and desumoylation assays, recombinant GST-SAE1/SAE2, GST-Ubc9, GST-SUM01 and GST-Mdm2 proteins expressed in Escherichia coli were affinity purified. The $20-\mu$ reaction buffer containing $50 \mathrm{mM}$ Tris- $\mathrm{HCl}$ ( $\mathrm{pH} 7.6), 5 \mathrm{mM}$ magnesium chloride, $10 \mathrm{mM}$ ATP, $1 \mu \mathrm{g}$ of GST-SAE1/2, $2 \mu \mathrm{g}$ of GST-Ubc9, $10 \mu \mathrm{g}$ of GST-SUMO1 and $200 \mathrm{ng}$ GST-Mdm2 with the presence of protease inhibitor cocktail was incubated for $3 \mathrm{~h}$ at $37^{\circ} \mathrm{C}$. The desumoylation reaction was then carried out in $10 \mu$ lof the above sumoylation mixture with the addition of purified MT-SENP2. After incubation overnight at $37^{\circ} \mathrm{C}$, the samples were analyzed by SDS-PAGE and immunoblot analysis of Mdm2 and SUMO1.

For desumoylation analysis using cell extracts, 293T cells were transfected with HA-SUMO1 plus or minus pCS2-SENP2, as well as HCT116, and HCT116-SENP2 cells were transfected with HA-SUMO1 using Lipofectamine 2000 (Invitrogen). After $48 \mathrm{~h}$, cell lysates were collected with buffer containing $100 \mathrm{mM}$ sodium chloride, $20 \mathrm{mM}$ Tris-HCl (pH 7.5), 1\% Triton X-100, protease inhibitor cocktail (Sigma-Aldrich), $1 \mathrm{mM}$ sodium molybdate, $1 \mathrm{mM}$ sodium vanadate and $1 \mathrm{mM}$ PMSF, followed by immunoprecipitation with anti-Mdm2 antibody and protein $\mathrm{G}$ agarose beads. The precipitates were analyzed by SDS-PAGE and immunoblot. To perform desumoylation analysis, cell lysates were collected from 293T cells transfected with pCS2SENP2 or pCS2, followed by purification with anti-MT antibody-mediated immunoprecipitation. The purified MT or MT-SENP2 protein was then incubated with cell lysates isolated from the HA-SUM01-transfected 293T cells with or without the presence of $10 \mathrm{mM} \mathrm{NEM}$ for $4 \mathrm{~h}$ at $37^{\circ} \mathrm{C}$, followed by immunoblot analysis.

\section{Conflict of interest}

The authors declare no conflict of interest.

Acknowledgements. We thank Ronald Hay, Bert Vogelstein and Michael O'Reilly for reagents, H-M Ivy Yu for technical assistance, and Anthony Mirando and Peter Keng for comments and suggestions. This work is supported by National Institutes of Health Grant CA106308 to WH.

1. Melchior F. SUMO - nonclassical ubiquitin. Annu Rev Cell Dev Biol 2000; 16: 591-626.

2. Schwartz DC, Hochstrasser M. A superfamily of protein tags: ubiquitin, SUMO and related modifiers. Trends Biochem Sci 2003; 28: 321-328.

3. Seeler JS, Dejean A. Nuclear and unclear functions of SUMO. Nat Rev 2003; 4: 690-699.

4. Muller S, Ledl A, Schmidt D. SUMO: a regulator of gene expression and genome integrity. Oncogene 2004; 23: 1998-2008.

5. Melchior F, Schergaut M, Pichler A. SUMO: ligases, isopeptidases and nuclear pores. Trends Biochem Sci 2003; 28: 612-618.

6. Gill G. SUMO and ubiquitin in the nucleus: different functions, similar mechanisms? Genes Dev 2004; 18: 2046-2059.

7. Chiu SY, Asai N, Costantini F, Hsu W. SUMO-specific protease 2 is essential for modulating p53-Mdm2 in development of trophoblast stem cell niches and lineages. PLoS Biol 2008; 6: e310.

8. Marine JC, Lozano G. Mdm2-mediated ubiquitylation: p53 and beyond. Cell Death Differ 2010; 17: 93-102.

9. Meek DW, Knippschild U. Posttranslational modification of MDM2. Mol Cancer Res 2003; 1: 1017-1026.

10. Melchior F, Hengst L. SUMO-1 and p53. Cell Cycle 2002; 1: 245-249.

11. Marine JC. p53 stabilization: the importance of nuclear import. Cell Death Differ 2010; 17: 191-192.

12. Storchova Z, Pellman D. From polyploidy to aneuploidy, genome instability and cancer Nat Rev 2004; 5: 45-54.
13. Lanni JS, Jacks T. Characterization of the p53-dependent postmitotic checkpoint following spindle disruption. Mol Cell Biol 1998; 18: 1055-1064.

14. Andreassen PR, Lohez OD, Lacroix FB, Margolis RL. Tetraploid state induces p53-dependent arrest of nontransformed mammalian cells in G1. Mol Biol Cell 2001; 12: 1315-1328.

15. Yates KE, Korbel GA, Shtutman M, Roninson IB, DiMaio D. Repression of the SUMOspecific protease Senp1 induces p53-dependent premature senescence in normal human fibroblasts. Aging Cell 2008; 7: 609-621.

16. Green DR, Kroemer G. Cytoplasmic functions of the tumour suppressor p53. Nature 2009; 458: $1127-1130$

17. Vazquez A, Bond EE, Levine AJ, Bond GL. The genetics of the p53 pathway, apoptosis and cancer therapy. Nat Rev Drug Discov 2008; 7: 979-987.

18. Polyak K, Waldman T, He TC, Kinzler KW, Vogelstein B. Genetic determinants of p53-induced apoptosis and growth arrest. Genes Dev 1996; 10: 1945-1952.

19. Fogal V, Gostissa M, Sandy P, Zacchi P, Sternsdorf T, Jensen K et al. Regulation of p53 activity in nuclear bodies by a specific PML isoform. EMBO J 2000; 19: $6185-6195$

20. Pearson M, Carbone R, Sebastiani C, Cioce M, Fagioli M, Saito S et al. PML regulates p53 acetylation and premature senescence induced by oncogenic Ras. Nature 2000; 406: 207-210.

21. Levine AJ, Oren M. The first 30 years of p53: growing ever more complex. Nat Rev Cancer 2009; 9: 749-758.

22. Vogelstein B, Lane D, Levine AJ. Surfing the p53 network. Nature 2000; 408: 307-310.

23. Vousden KH, Lane DP. p53 in health and disease. Nat Rev 2007; 8: 275-283.

24. Meek DW. Tumour suppression by p53: a role for the DNA damage response? Nat Rev Cancer 2009; 9: 714-723.

25. Riley $\mathrm{T}$, Sontag $\mathrm{E}$, Chen $\mathrm{P}$, Levine A. Transcriptional control of human p53-regulated genes. Nat Rev 2008; 9: 402-412.

26. Kruse JP, Gu W. Modes of p53 regulation. Cell 2009; 137: 609-622.

27. Morris JR, Boutell C, Keppler M, Densham R, Weekes D, Alamshah A et al. The SUMO modification pathway is involved in the BRCA1 response to genotoxic stress. Nature 2009; 462: 886-890.

28. Galanty Y, Belotserkovskaya R, Coates J, Polo S, Miller KM, Jackson SP. Mammalian SUMO E3-ligases PIAS1 and PIAS4 promote responses to DNA double-strand breaks. Nature 2009; 462: 935-939.

29. Li FP, Fraumeni Jr JF. Soft-tissue sarcomas, breast cancer, and other neoplasms. A familial syndrome? Ann Intern Med 1969; 71: 747-752.

30. Dawlaty MM, Malureanu L, Jeganathan KB, Kao E, Sustmann C, Tahk S et al. Resolution of sister centromeres requires RanBP2-mediated SUMOylation of topoisomerase Ilalpha. Cell 2008; 133: 103-115.

31. Kaikkonen S, Jaaskelainen $T$, Karvonen U, Rytinki MM, Makkonen $H$, Gioeli D et al. SUMO-specific protease 1 (SENP1) reverses the hormone-augmented SUMOylation of androgen receptor and modulates gene responses in prostate cancer cells. Mol Endocrinol (Baltimore, Md) 2009; 23: 292-307.

32. Tatham MH, Jaffray E, Vaughan OA, Desterro JM, Botting $\mathrm{CH}$, Naismith JH et al. Polymeric chains of SUMO-2 and SUMO-3 are conjugated to protein substrates by SAE1/SAE2 and Ubc9. J Biol Chem 2001; 276: 35368-35374.

33. Desterro JM, Rodriguez MS, Hay RT. SUMO-1 modification of IkappaBalpha inhibits NF-kappaB activation. Mol Cell 1998; 2: 233-239.

34. Fu J, Jiang M, Mirando AJ, Yu HM, Hsu W. Reciprocal regulation of Wnt and Gpr177/ mouse Wntless is required for embryonic axis formation. Proc Natl Acad Sci USA 2009; 106: $18598-18603$

35. Liu B, Yu HM, Hsu W. Craniosynostosis caused by Axin2 deficiency is mediated through distinct functions of beta-catenin in proliferation and differentiation. Dev Biol 2007; 301: 298-308.

36. Liu B, Yu HM, Huang J, Hsu W. Co-opted JNK/SAPK signaling in Wnt/beta-catenininduced tumorigenesis. Neoplasia (New York, NY) 2008; 10: 1004-1013.

37. Maruyama T, Mirando AJ, Deng CX, Hsu W. The balance of WNT and FGF signaling influences mesenchymal stem cell fate during skeletal development. Sci Signal 2010; 3: ra40.

38. Yu HM, Liu B, Costantini F, Hsu W. Impaired neural development caused by inducible expression of Axin in transgenic mice. Mech Dev 2007; 124: 146-156.

39. Yu HM, Jin Y, Fu J, Hsu W. Expression of Gpr177, a Wnt trafficking regulator, in mouse embryogenesis. Dev Dyn 2010; 239: 2102-2109.

40. Yu HM, Jerchow B, Sheu TJ, Liu B, Costantini F, Puzas JE et al. The role of Axin2 in calvarial morphogenesis and craniosynostosis. Development (Cambridge, England) 2005; 132: 1995-2005. 